\title{
Intrahepatic bile duct variation: MR cholangiography and implication in hepatobiliary surgery
}

\author{
Mona El Hariri ${ }^{1 *}$ (D) and Mohamed M. Riad ${ }^{2}$
}

\begin{abstract}
Background: The aim of this study was to assess the prevalence of biliary anatomical variants using 3-T MR cholangiography (MRC) with its impact in reduction of the complication of hepatobiliary surgical techniques.

Results: MRC was applied to 120 subjects (24 potential liver donors and 96 volunteers) and the right posterior hepatic duct insertion was documented, and accordingly, the biliary variants were classified based on Huang classification (Huang et al, Transplant Proc 28: 1669-1670, 1996).

Biliary anatomic variants were divided based on Huang classification: Huang A1, 65.83\% $(n=79)$; Huang A2, $11.67 \%(n=14)$; Huang A3, 13.3\% $(n=16)$; Huang A4, 7.5\% $(n=9)$; and Huang A5, 1.67\% $(n=2)$. The total frequency for A2, A3, A4, and A5 was $34.17 \%(n=41)$. The distance between RPHD insertion and the junction of right and left hepatic ducts $(L)$ was measured, and Huang A1 cases were then subtyped into $S 1$ subtype $(L>1 \mathrm{~cm})$ and $S 2$ subtype $(L \leq 1 \mathrm{~cm})$. We had 52 subjects with subtype $\mathrm{S} 1$ (43.33\%) and 27 subjects with subtype S2 (22.5\%).

Twenty-three subjects had bile duct exploration or intraoperative cholangiograms and showed Huang type A1 in $14(60.87 \%)$, type A2 in 3 (13.05\%), and type A3 in 6 (26.08\%). Twenty-two (95.65\%) had the same classification in MRC and intraoperative while only one case (4.35\%) was considered as A2 at MRC but the intraoperative classification was Huang $A 3$, which was attributed to the insertion of the RPHD insertion at the distal end of the left hepatic duct.
\end{abstract}

Conclusion: MRC is an accurate tool for biliary tract mapping before hepatobiliary surgery to provide excellent identification of biliary variants which can reduce the incidence of biliary complications.

\section{Background}

Detailed mapping of biliary anatomy is an essential preoperative requirement for the proper choice of therapeutic approach as well as for reduction of iatrogenic biliary pathology which can negatively affect the hepatobiliary surgery outcome [1-4].

Despite the gross improvement in the surgical techniques of liver transplantation and the better survival rates, the biliary complications is still on the top of the complication in living donor liver transplantation, occurring in $7-10 \%$ of donors; also, biliary complication has a prevalence of $3.6-8.1 \%$ after hepatic tumor

\footnotetext{
* Correspondence: Doctormona2000@yahoo.com

'Department of Radiodiagnosis, Faculty of Medicine, Zagazig University, PO. BOX 184, Zagazig, Sharkia 44511, Egypt

Full list of author information is available at the end of the article
}

resection [5-8]. Even in low complication rate procedure such as laparoscopic cholecystectomy (complications $<1 \%$ ), the pre-operative biliary mapping can avoid iatrogenic biliary injuries of non-recognized anatomical variants [9].

Biliary mapping using the diagnostic endoscopic retrograde cholangiography has major complication ranging from 1.4 to $3.2 \%$, so having a non-invasive, simpler, and more safe technique would be of great value [10-12].

The start of using magnetic resonance cholangiopancreatography (MRCP) in early 1990s played a nonradiated, non-invasive safe modality $[9,10,13]$ with more advancement, and using 3-T clinical MRI, MRC showed a highly promising tool for biliary tree 
assessment that can display a nearly motion artifact-free high-resolution images in short scan time [14].

There are many classifications of biliary anatomical variations, of which Huang classification method [15] is a widely used based on the variable insertion of right posterior hepatic bile duct (RPHD). It has five variants classification (Table 1). While Champetier classification [16] excluded the dominant Huang type $\mathrm{A}$ and dealt with other variants only, it has one more type (E); in that type, cystic duct receives the opening of both right anterior hepatic duct (RAHD) and RPHD.

The aim of the current study was the assessment of the prevalence of the anatomical intrahepatic biliary variants by using 3-T MR cholangiography (MRC) with its impact to lower hepatobiliary surgical techniques' complications.

\section{Methods}

\section{Study subjects}

The current study included 120 subjects: 24 potential liver donors and 96 volunteers (referred for MRI lumbar spine) and had no medical history of significant problems. They were 62 males and 58 females. The ages ranged from 18 to 63 years (mean $41 \pm 16.3$ years).

Approval from our institutional review board was obtained, and subjects' consent was taken.

\section{MRC protocol}

MRI was done for all subjects using 3.0-T magnetic resonance system (Intera Achieva; Philips-Netherlands). A six-element phased array coil was used.

The subjects were instructed to fast $6 \mathrm{~h}$ prior to MRI to distend the gallbladder and to have an empty stomach as well as to suppress the movement of intestines; however, no anti-peristaltic medicine or oral contrast media were applied.

- The protocol of MRI included routine transverse T1-WI breath-hold in- and opposed-phase gradientecho MRI as well as T2-WI TSE MRI with fat saturation and MRC:

- Coronal and coronal oblique $\left( \pm 15^{\circ}\right)$ breath-hold single-slice RARE (rapid acquisition with relaxation enhancement).

Table 1 Huang classification

\begin{tabular}{ll}
\hline Variant type & Site of opening of tight posterior hepatic duct (RPHD) \\
\hline Huang A1 & The right anterior hepatic duct (RAHD) \\
Huang A2 & The hepatic confluence (trifurcation) \\
Huang A3 & The left hepatic duct (LHD) \\
Huang A4 & The main hepatic duct (MHD) \\
Huang A5 & The cystic duct \\
\hline
\end{tabular}

- Then, respiratory-triggered 3D TSE (turbo spin-echo) was obtained. Abdominal respiratory belt was used.

Image data analysis was done using dedicated workstation depending on the raw images, and reconstruction was done to obtain cholangiographic images with a MIP (maximum intensity projection) and VR (volume rendering) sometimes (Table 2).

\section{Image interpretation}

The insertion of RPHD was documented in every subject; accordingly, the biliary variants were grouped according to Huang classification [15]. The distance between insertion of RPHD and the junction of right and left hepatic duct was measured, and then the mean for each variant was estimated.

Comparison of the results of cholangiograms done intraoperatively as well as explorations of bile duct results $(n=22$ donor) were compared with the corresponding MRC results.

\section{Statistical analysis}

Statistical package SPSS version 17 was used for analysis of statistics. McNemar's test and $t$ test were applied for statistical analyses ( $P$ value of $<0.05$ was assigned to be a significant).

\section{Results}

The current study included 120 subjects (62 males and 58 females) with age ranged from 18 to 63 years (mean $41 \pm$ 16.3 years).

According to RPHD insertion [15] (Fig. 1), biliary anatomic variants were divided based on Huang classification.

Accordingly, the prevalence of variants was as follows (Fig. 2 and Table 3):

Type A1 (Fig. 3), 65.83\% ( $n=79)$; type A2 (Fig. 4), $11.67 \%(n=14)$; Huang A3 (Fig. 5), 13.3\% $(n=16)$;

Table $2 \mathrm{MRI}$ protocol

\begin{tabular}{|c|c|c|}
\hline & RARE & 3D-TSE \\
\hline $\begin{array}{l}\text { TR (repetition } \\
\text { time) }\end{array}$ & $9800 \mathrm{~ms}$ & $2600 \mathrm{~ms}$ \\
\hline TE (echo time) & $920 \mathrm{~ms}$ & $740 \mathrm{~ms}$ \\
\hline Matrix & $256 \times 256$ & $217 \times 256$ \\
\hline $\begin{array}{l}\text { Slice thickness } \\
(\mathrm{mm})\end{array}$ & 50 & 1 \\
\hline Flip angle & $90^{\circ}$ & $90^{\circ}$ \\
\hline Field of view & $30 \times 30 \mathrm{~cm}^{2}$ & $30 \times 30 \mathrm{~cm}^{2}$ \\
\hline Echo train length & 256 & 87 \\
\hline $\begin{array}{l}\text { Signal averages } \\
\text { number }\end{array}$ & 1 & 1 \\
\hline Acquisition time & $\begin{array}{l}\text { Each slice: } \\
9.8 \mathrm{~s}\end{array}$ & $\begin{array}{l}\text { Depend on the respiratory frequency } \\
(2-5 \mathrm{~min})\end{array}$ \\
\hline
\end{tabular}




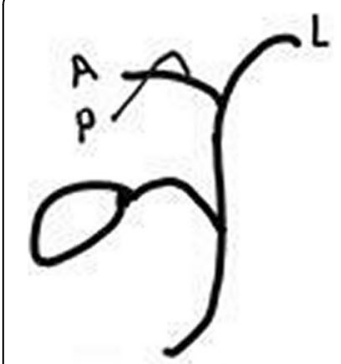

Huang A1

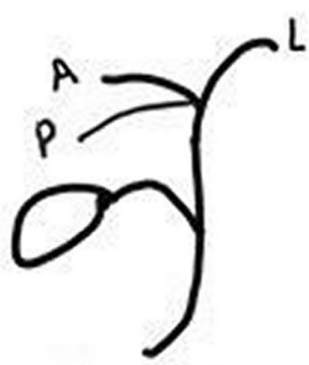

Huang A2

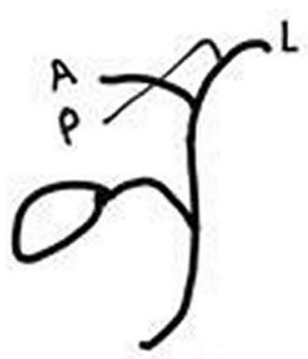

Huang A3

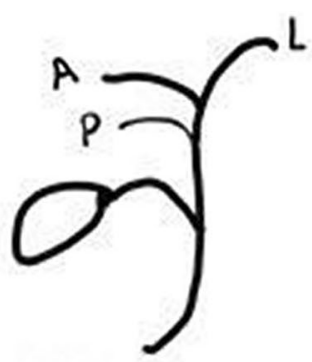

Huang A4

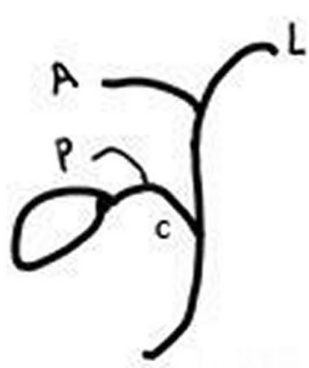

Huang A5

\section{$A=R A H D, P=R P H D, L=L H D, C=$ cystic duct}

Fig. 1 Diagram illustrates Huang classification (RPHD, right posterior hepatic duct; RAHD, right anterior hepatic duct; LHD, Left hepatic duct)

Huang A4 (Fig. 6), 7.5\% ( $n=9)$; and type A5 (Fig. 7), $1.67 \%(n=2)$.

The total frequency for A2, A3, A4, and A5 collectively was $34.17 \%(n=41)$.

The distance between the insertion of RPHD and the right and left hepatic ducts' junction (L) has a surgical importance as if $\mathrm{L}$ is more than $1 \mathrm{~cm}$, it may need modification of surgical techniques. So, we had to subtype Huang A1 cases into $\mathrm{S} 1$ subtype $(\mathrm{L}>1 \mathrm{~cm})$ and $\mathrm{S} 2$ subtype $(\mathrm{L} \leq 1$ $\mathrm{cm})$. Our subjects were 52 with $\mathrm{S} 1$ (43.33\%) subtype and 27 with S2 (22.5\%) subtype.

The mean distance between the RPHD and the right and left hepatic ducts' junction was $9.73 \pm 5.02 \mathrm{~mm}$ (ranging from 4 to $24 \mathrm{~mm}$ ) for Huang $\mathrm{A} 1$ and $9.35 \pm 4.93$ $\mathrm{mm}$ (range $=3.4-22.3 \mathrm{~mm}$ ) for Huang A3, while it was $9.16 \pm 4.67$ (ranging from 4.2 to $22.9 \mathrm{~mm}$ ) in Huang A4.

The comparison of these different Huang types was insignificant regarding the distances between the insertion of RPHD insertion to the junction of the right and left hepatic ducts.

Twenty-three subjects had bile duct exploration or intraoperative cholangiograms and showed Huang A1 in 14 (60.87\%), Huang A2 in 3 (13.05\%), and Huang A3 in 6 (26.08\%). Twenty-two (95.65\%) had the same classification in MRC and intraoperative while only one case (4.35\%) was considered as A2 at MRC but the intraoperative classification was Huang A3, which was attributed to the insertion of the RPHD insertion at the distal end of the left hepatic duct.

\section{Discussion}

The need for precise intrahepatic biliary anatomy is essential especially with the biliary intervention procedures as well as liver surgery including liver resection and transplantation to have a safe hepatectomy and reduce biliary complications [17-20].

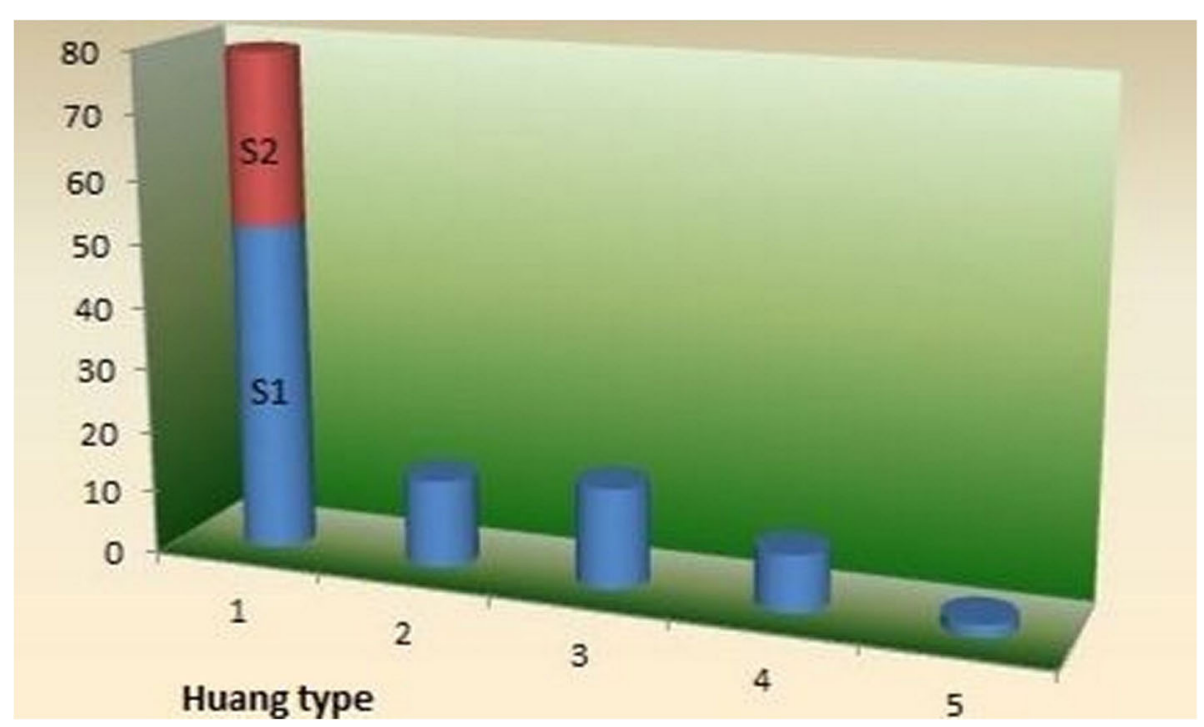

Fig. 2 Prevalence of biliary variants (Huang types) among study subjects 
Table 3 Anatomical biliary variants prevalence

\begin{tabular}{lll}
\hline Type & Number & Percent \\
\hline Huang A 1 & 79 & 65.83 \\
Huang A 1 S1 & 52 & 43.33 \\
Huang A 1 S2 & 27 & 22.5 \\
Huang A 2 & 14 & 11.67 \\
Huang A 3 & 16 & 13.33 \\
Huang A 4 & 9 & 7.5 \\
Huang A 5 & 2 & 1.67 \\
\hline
\end{tabular}

While biliary anatomical variants are not a contraindication for liver donation, however, detailed accurate pre-operative identification is essential to avoid iatrogenic ligation of the donor or recipient's major biliary tract, for example, ligation of aberrant RAHD or RPHD drainage into the left hepatic duct can cause cirrhosis $[18,19]$. On the other hand, during right lobe transplantation, multiple biliary anastomoses in the recipient may be needed to prevent biliary obstruction [21].

MRCP is a noninvasive diagnostic technique with no radiation hazards and avoids the hazards of nephrotoxic contrast media and ERCP. It can show the high signal of the biliary and pancreatic secretions with dark background (sensitivity up to 90\%) in normal biliary mapping [22, 23].

Breath-hold imaging can eliminate the artifacts caused by respiratory motion with the ability to improve spatial resolution by using a longer time of acquisition. It shows the advantage of a relatively short time of acquisition; however, the quality of images is affected by a low signal-to-noise ratio as well as low spatial resolution. The respiratory-triggered technique is able to extend the time of acquisition thus having higher spatial resolution [24].

There is a high prevalence of biliary variants which was shown in a many previous studies [25-27].

In the current study, we used Huang classification to categorize intrahepatic ducts according to the RPHD insertion.

The current study showed that vast subject number had intrahepatic duct Huang type A1 (typical type) representing $65.83 \%(n=79)$ of the examined subjects followed by Huang A2, $11.67 \%(n=14)$; Huang A3, $13.3(n=16)$; type A4, $7.5 \%(n=9)$; and Huang type A5, $1.67(n=2)$.

This coincides with many previous studies [3, 15, 24, 28-33] while lower incidence of A1 (56\%) was encountered in the study of Wang et al. [34].

Huang type $\mathrm{A}$ is optimum for right hepatic lobe living transplantation as it is simple; however, the right hepatic duct (RHD) length has a crucial impact as with sufficient length, one biliary-enteric anastomosis may be done easily, while in the case of short RHD, it may need modification as double anastomoses to avoid injury risk of the bile duct in hepatic resection $[15,19,20,35]$. In the current study, Huang A1 was the dominant type representing $65.83 \%(n=79)$ of the subjects included in our study. Due to the surgical

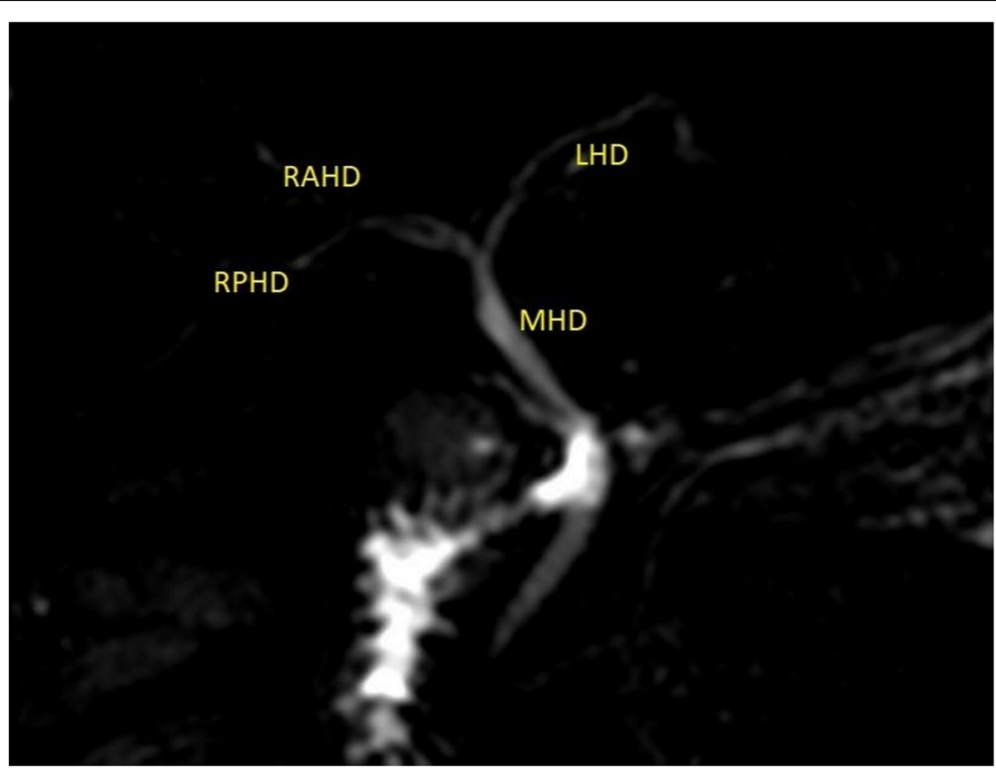

Fig. 3 MRC (RARE) Huang type A1: RPHD drain into the RAHD (RPHD, right posterior hepatic duct; RAHD, right anterior hepatic duct; LHD, left hepatic duct; and MHD, main hepatic duct) 

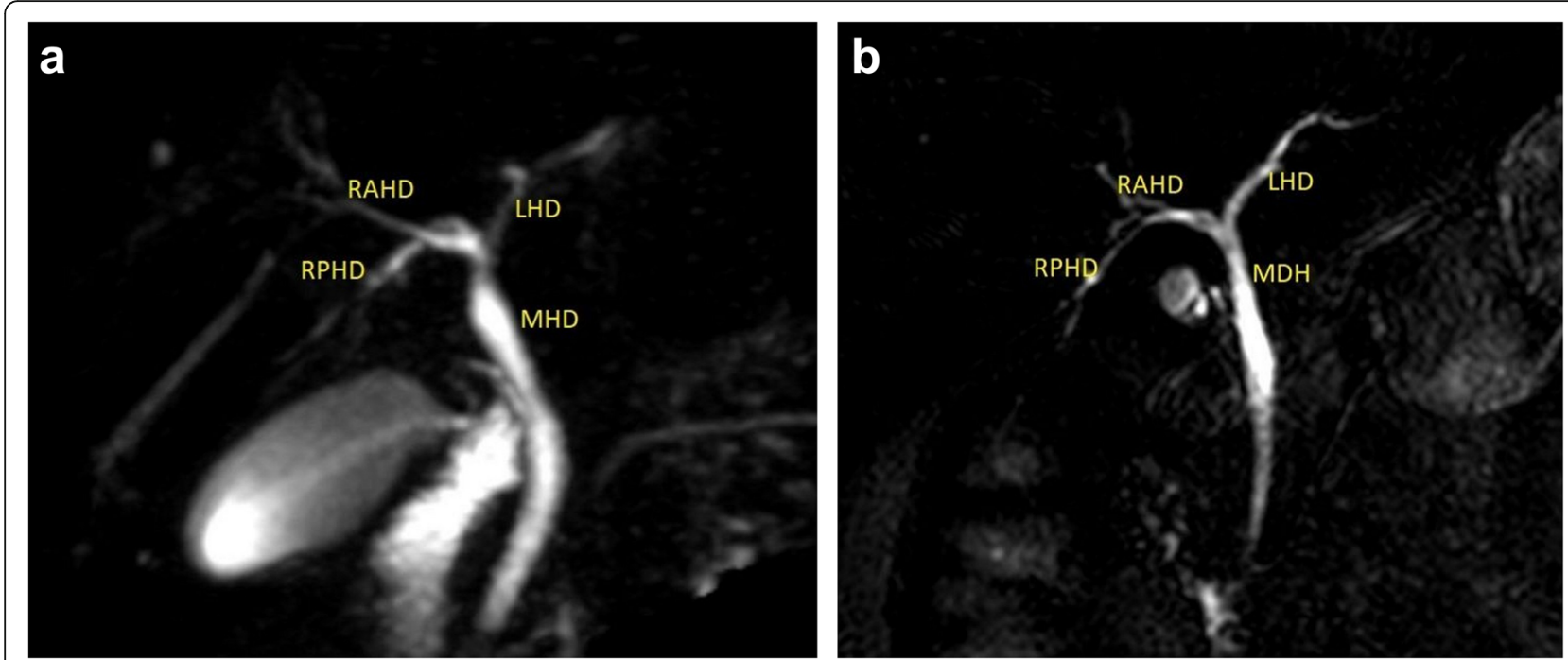

Fig. 4 MRC 3D TSE (a) and MRC (RARE) (b). Trifurcation (Huang A2): RPHD open into the hepatic common confluence (RPHD, right posterior hepatic duct; RAHD, right anterior hepatic duct; LHD, left hepatic duct; and MHD, main hepatic duct)

importance of the distance between RPHD insertion and the right and left hepatic duct junction which assume a trifurcation pattern (common RAHD, RHPD, and LHD junction) for distance of $1 \mathrm{~cm}$ or less [20, $28,35,36]$, we had to subtype our subjects of Huang A1 based on the distance (L) between the insertion of RPHD and the right and left hepatic duct junction into $\mathrm{S} 1(\mathrm{~L}>1 \mathrm{~cm})$ and $\mathrm{S} 2(\mathrm{~L}=1 \mathrm{~cm}$ or less). Accordingly, we had Huang A1 subtypes: subtype S1 $(n=52$, $43.33 \%)$ and subtype S2 $(n=27,22.5 \%)$.

In current study, the second predominant type was Huang type A3 in which the RPHD ends into the LHD, and it was seen in $13.3 \%(n=16)$ which coincides with many previous studies $[3,15,20,29,31,36]$. Also, it is close to the results of Basaran et al. [24] (Gawad 22) and Wang et al. [34] which was $20 \%$ and $18 \%$ respectively.

This variant may cause donor biliary injury and may necessitate double anastomoses to prevent postoperative biliary complication such as biliary leakage or segmental atrophy $[9,20,36]$.

The third frequency was in the current study was for Huang A2 in which the RPHD open to the hepatic confluence (trifurcation) and represented $11.67 \%(n=14)$ of our subjects which is near to the results of Wang et al. [34] which was $11 \%$ and higher than the frequency in the series of Basaran et al. [24] which was 5\%. Some centers may avoid graft harvesting in biliary trifurcation to prevent a higher rate of postoperative complications.
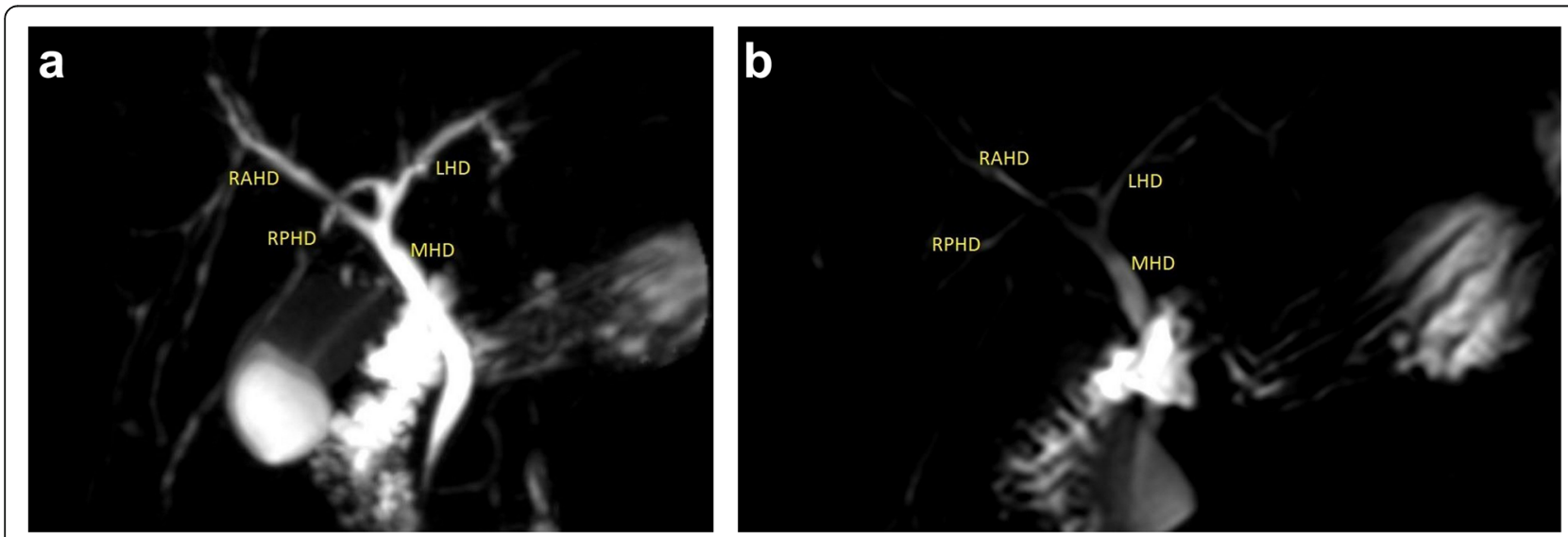

Fig. 5 MRC 3D TSE (a) and MRC (RARE) (b). Huang type A3: RPHD opens into LHD (RPHD, right posterior hepatic duct; RAHD, right anterior hepatic duct; LHD, left hepatic duct; and MHD, main hepatic duct) 


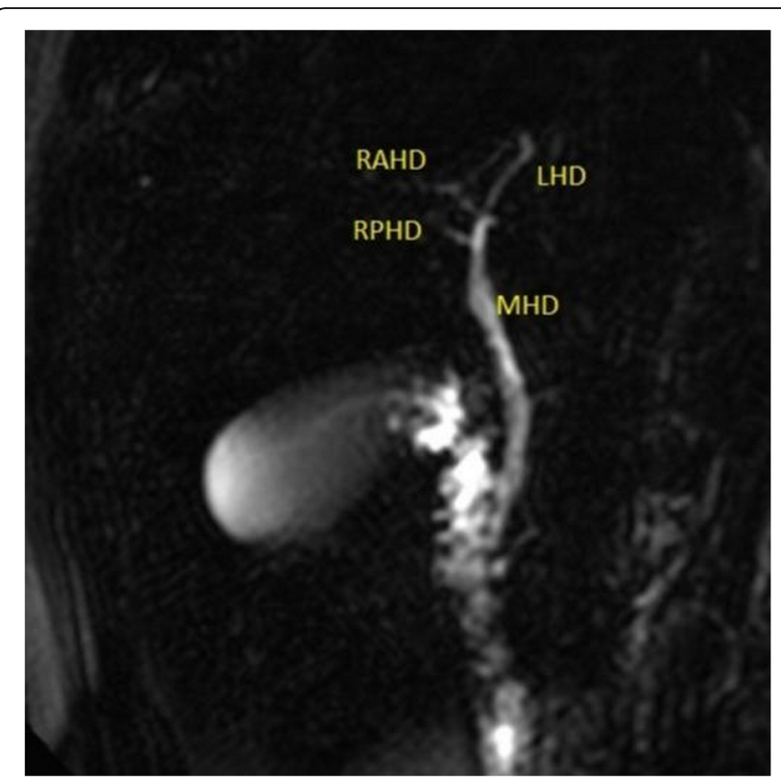

Fig. 6 MRC (RARE). Huang type A4: RPHD opens into main hepatic duct (MHD) (RPHD, right posterior hepatic duct; RAHD, right anterior hepatic duct; LHD, left hepatic duct; and MHD, main hepatic duct)

In Huang types $\mathrm{A} 4$ and $\mathrm{A} 5$, there is aberrant right posterior hepatic duct that drains to the CHD or cystic ducts respectively this can be inadvertently ligated or injured during biliary surgery [24].

Double anastomoses may be needed in Huang type A4 also to prevent possible postoperative biliary complications in cases of liver transplantation [19, 20]. In current report, Huang A4 was seen in $7.5 \%$ of current subjects $(n=9)$ which is near to the results of Wang et al. [34] and higher than the results of Basaran et al. [24] who had donors having their RPHD drain into the main duct in $8 \%$ and $2.5 \%$ respectively.

We encountered Huang A5 in two subjects (1.67\%) which is nearly of similar incidence reported in many previous studies [20,37, 38].

Huang type 5 with RPHD draining into the cystic duct is of biliary surgical importance especially during laparoscopy as its damage may lead to biliary leakage and biloma [20, 37, 38].

In current study, 23 subjects had bile duct exploration or intraoperative cholangiograms, and 22 (95.65\%) of them had the same MRC Huang type while only one case (4.35\%) was reported as A2 at MRC but shown at intraoperative classification as Huang A3 due to RPHD insertion into the left hepatic duct's distal end.

We had some limitation in our study; first, it was a small cohort study, and secondly, only 23 of our subjects were confirmed by intraoperative procedure.

\section{Conclusion}

MRC is an accurate tool for biliary tract mapping before hepatobiliary surgery to provide excellent identification of biliary anatomical variants which can diminish the possibility of biliary complications.

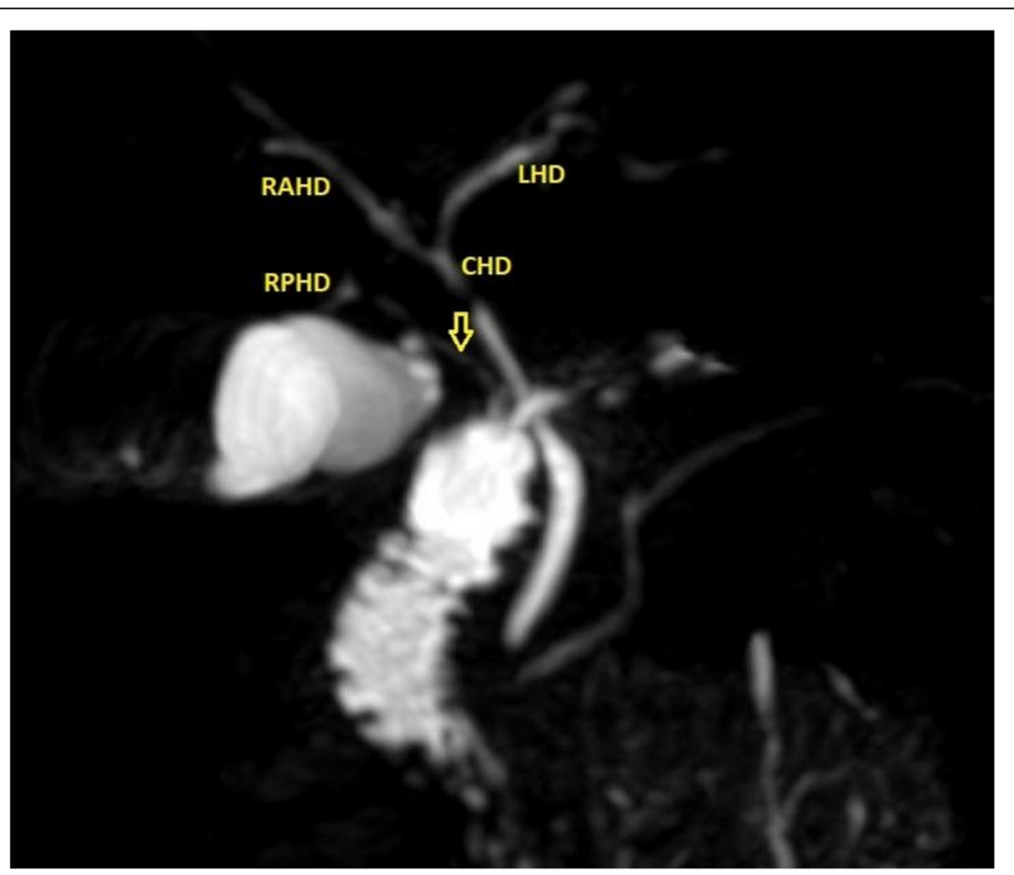

Fig. 7 MRC 3D TSE. Huang type A5: RPHD drains into cystic duct (arrow) (RAHD, right anterior hepatic duct; LHD, left hepatic duct; MHD, main hepatic duct) 


\section{Abbreviations}

ERCP: Endoscopic retrograde cholangiopancreatography; LHD: Left hepatic duct; MIP: Maximum intensity projection; MRC: Magnetic resonance cholangiography; MRCP: Magnetic resonance cholangiopancreatography; MRI: Magnetic resonance imaging; RAHD: Right anterior hepatic duct; RPHD: Right posterior hepatic duct; VR: Volume rendering

\section{Acknowledgements}

Not applicable

\section{Authors' contributions}

EM conceived of the study, participated in its design and coordination, drafted the manuscript, and carried out the radiological results. RM helped in the drafting of the results and participated in the surgical assessment. Both authors read and approved the final manuscript.

\section{Funding}

Not applicable.

\section{Availability of data and materials}

The data that support the findings of this study are available on request from the corresponding author.

\section{Ethics approval and consent to participate}

This study was approved by the Ethics Committee of Faculty of Medicine, Zagazig University (study protocol was approved in January 2017). The committee has no reference number, only the date. An informed written consent from each patient was taken before enrollment into the study.

\section{Consent for publication}

A written informed consent was obtained from all individuals relevant to this research.

\section{Competing interests}

The authors declare that they have no competing interests.

\section{Author details}

'Department of Radiodiagnosis, Faculty of Medicine, Zagazig University, PO. BOX 184, Zagazig, Sharkia 44511, Egypt. 'Department of General Surgery, Faculty of Medicine, Zagazig University, Zagazig, Egypt.

Received: 25 November 2019 Accepted: 29 November 2019

Published online: 12 December 2019

\section{References}

1. Ragozzino A, De Ritis R, Mosca A, laccarino V, Imbriaco M (2004) Value of MR cholangiography in patients with iatrogenic bile duct injury after cholecystectomy. AJR Am J Roentgenol 183:1567-1572

2. Guiney MJ, Kruskal JB, Sosna J, Hanto DW, Goldberg SN, Raptopoulos V (2003) Multi-detector row CT of relevant vascular anatomy of the surgical plane in split-liver transplantation. Radiology 229:401-407

3. Kapoor V, Peterson M, Baron R, Patel S, Eghtesad B, Fung J (2002) Intrahepatic biliary anatomy of living adult liver donors: correlation of Mangafodipir Trisodium-enhanced MR cholangiography and intraoperative cholangiography. AJR 179:1281-1286

4. Lau WY, Lai ECH (2007) Classification of iatrogenic bile duct injury. Hepatobiliary Pancreat Dis Int 6:459-463

5. Itamoto T, Emoto K, Mitsuta H, Fukuda S, Ohdan H, Tashiro H, Asahara T (2006) Safety of donor right hepatectomy for adult-to-adult living donor liver transplantation. Transpl Int 19(3):177-183

6. Valls C, Alba E, Cruz M, Figueras J, Andía E, Sanchez A, Llado L, Serrano T (2005) Biliary complications after liver transplantation: diagnosis with MR cholangiopancreatography. AJR Am J Roentgenol 184:812-820

7. Capussotti L, Ferrero A, Vigano L, Sgotto E, Muratore A, Polastri R (2006) Bile leakage and liver resection: where is the risk? Arch Surg 141(7):690-694

8. Pecchi A, De Santis M, Di Benedetto F, Gibertini M, Gerunda G, Torricelli P (2010) Role of magnetic resonance cholangiography in biliary complications of orthotopic liver transplantation. Radiol Med 115:1065-1079

9. Catalano OA, Singh AH, Uppot RN, Hahn PF, Ferrone CR, Sahani DV. Vascular and biliary variants in the liver: implications for liver surgery RadioGraphics 2008; 28:359-378
10. Yeh M, Breiman RS, Taouli B, Qayyum A, Roberts JB, Coakley FV (2004) Biliary tract depiction in living potential liver donors: comparison of conventional MR, Mangafodipir Trisodium-enhanced excretory MR, and multi-detector row CT cholangiography_initial experience. Radiology 230:645-651

11. Goyen M, Schroeder T, Ruehm S, Debatin F (2004) MR-based assessment of potential living related liver donors. Eur Radiol Suppl 14(7):36-40

12. Masci E, Toti G, Mariani A, Curioni S, Lomazzi A, Dinelli M, Minoli G, Crosta C, Comin U, Fertitta A, Prada A, Passoni GR, Testoni PA. Com. Complications of diagnostic and therapeutic ERCP: a prospective multicenter study. Am J Gastroenterol 2001; 96:417-423

13. Delaney L, Applegate KE, Karmazyn B, Akisik F, Jennings G (2008) MR cholangiopancreatography in ;children: feasibility, safety, and initial experience. Pediatr Radiol 38:64-75

14. Lee WK, Shannon SP (2007) 3-tesla magnetic resonance imaging (MRI)- is it ready for prime time clinical applications? Can J Med Radiat Technol:37-50

15. Huang TL, Cheng YF, Chen CL, Lee TY (1996) Variants of the bile ducts: clinical application in the potential donor of livingrelated hepatic transplantation. Transplant Proc 28:1669-1670

16. Champetier J (1994) Les voies biliaires. In: Chevrel JP (ed) Anatomie clinique, Le tronc. Springer, Paris, p 416

17. Park CH, Cho HJ, Kwack EY, Choi CS, Kang IW, Yoon JS (1991) Intrahepatic biliary duct anatomy and its variations. J Korean Radiol Soc 27:827-831

18. Goldman J, Florman S, Varotti G, Gondolesi GE, Gerning A, Fishbein T, Kim L, Schwartz ME. Noninvasive preoperative evaluation of biliary anatomy in right-lobe living donors with mangafodipir trisodium enhanced

19. Mortele KJ, Ros PR (2001) Anatomic variants of the biliary tree: MR cholangiographic findings and clinical applications. AJR Am J Roentgenol 177:389-394 MR cholangiography. Transplant Proc 2003;35(4):1421-2

20. Choi JW, Kim TK, Kim KW, Kim AY, Kim PN, Ha HK, Lee MG (2003) Anatomic variation in intrahepatic bile ducts: an analysis of intraoperative cholangiograms in 300 consecutive donors for living donor liver transplantation. Korean J Radiol 4(2):85-90

21. Caruso S, Miraglia R, Maruzzelli L, Gruttadauria S, Luca A, Gridelli B (2009) Imaging in liver transplantation. World J Gastroenterol 15(6):675-683

22. Glockner JF (2007) Hepatobiliary MRl: current concepts and controversies. J Magn Reson Imaging 25(4):681-695

23. Catalano OA, Singh AH, Uppot RN, Hahn PF, Ferrone CR, Sahani DV (2008) Vascular and biliary variants in the liver: implications for liver surgery. Radiographics 28(2):359-378

24. Basaran C, Agildere AM, Donmez FY, Sevmis S, Budakoglu I, Krakayali H, Habera M (2008) MR cholangiopancreatography with T2- weighted prospective acquisition correction turbo spin-echo sequence of the biliary anatomy of potential living liver transplant donors. AJR Am J Roentgenol 190(6):1527-1533

25. Couinaud C (1989) The surgical anatomy of the liver revisited. Maugein \& Cie, Paris

26. Smadja C, Blumgart LH (1994) The biliary tract and the anatomy of biliary exposure, 2nd edn. Churchill Livingstone, Edinburgh, pp 11-24

27. Schroeder T, Malago M, Debatin JF, Testa G, Nadalin S, Broelsch CE et al (2002) Multidetector computed tomographic cholangiography in the evaluation of potential living liver donors. Transplantation 73:1972-1973

28. Ohkubo M, Nagino M, Kamiya J, Yuasa N, Oda K, Arai T et al (2004) Surgical anatomy of the bile ducts at the hepatic hilum as applied to living donor liver transplantation. Ann Surg 239:82-86

29. Kim RD, Sakamoto S, Haider MA, Molinari M, Gallinger S, McGilvray ID, et al. Role of magnetic resonance cholangiography in assessing biliary anatomy in right lobe living donors. Transplantation 200; 79:1417-1421

30. Dusunceli E, Erden A, Erden I (2004) Anatomic variations of the bile ducts: MRCP findings. Tani Girisim Radyol 10:296-303

31. Kitami M, Takase K, Murakami G, Ko S, Tsuboi M (2006) Types and frequencies of biliary tract variations associated with a major portal venous anomaly: analysis with multi-detector row $\mathrm{CT}$ cholangiography. Radiology 238:156-166

32. Cheng YF, Huang TL, Chen CL, Chen YS, Lee TY (1997) Variations of the intrahepatic bile ducts: application in living related liver transplantation and splitting liver transplantation. Clin Transpl 11:337-340

33. Turner MA, Fulcher AS (2001) The cystic duct: normal anatomy and disease processes. RadioGraphics 21:3-22

34. Wang ZJ, Yeh BM, Roberts JP, Breiman RS, Qayyum A, Coakley FV (2005) Living donor candidates for right hepatic lobe transplantation: evaluation at CT cholangiography-initial experience. Radiology 235(3):899-904 
35. Karakas HM, Celik T, Alicioglu B (2008) Bile duct anatomy of the Anatolian Caucasian population: Huang classification revisited. Surg Radiol Anat 30: 539-545

36. Limanond P, Raman SS, Ghobrial RM, Busuttil RW, Lu DSK (2004) The utility of MRCP in preoperative mapping of biliary anatomy in adult-to adult living related liver transplant donors. J Magn Reson Imaging 19:209-215

37. Ozsoy M, Zeytunlu M, Kilic M, Alper M, Sozbilen M The results of vascular and biliary variations in Turks liver donors: comparison with others. Int Scholarly Res Netw Surg. https://doi.org/10.5402/2011/367083

38. Champetier J, Letoublon C, Alnaasan I, Charvin B (1991) The cysticohepatic ducts: surgical implications. Surg Radiol Anat 13:203-211

\section{Publisher's Note}

Springer Nature remains neutral with regard to jurisdictional claims in published maps and institutional affiliations.

Submit your manuscript to a SpringerOpen ${ }^{\odot}$ journal and benefit from:

- Convenient online submission

Rigorous peer review

- Open access: articles freely available online

High visibility within the field

- Retaining the copyright to your article

Submit your next manuscript at $\boldsymbol{\nabla}$ springeropen.com 